\title{
New Type of Degenerate Poly-Frobenius-Euler Polynomials and Numbers
}

\author{
Waseem A. Khan
}

Department of Mathematics and Natural Sciences, Prince Mohammad Bin Fahd University, P.O Box 1664, Al Khobar 31952, Saudi Arabia

E-mail: wkhan1@pmu.edu.sa

Abstract. Motivated by Kim-Kim [19] introduced the new type of degenerate polyBernoulli polynomials by means of the degenerate polylogarithm function. In this paper, we define the degenerate poly-Frobenius-Euler polynomials, called the new type of degenerate poly-Frobenius-Euler polynomials, by means of the degenerate polylogarithm function. Then, we derive explicit expressions and some identities of those numbers and polynomials.

Keywords: polylogarithm function; Frobenius-Euler polynomials; new type of degenerate poly-Frobenius-Euler polynomials

2010 Mathematics Subject Classification: 05A19, 11B68, 11B83, 11 B95.

\section{Introduction}

Throughout this presentation, we use the following standard notions $\mathbb{N}=\{1,2, \cdots\}$, $\mathbb{N}_{0}=\{0,1,2, \cdots\}=\mathrm{N} \cup\{0\}, \mathbb{Z}^{-}=\{-1,-2, \cdots\}$. Also as usual $\mathbb{Z}$ denotes the set of integers, $\mathbb{R}$ denotes the set of real numbers and $\mathbb{C}$ denotes the set of complex numbers.

The classical Bernoulli $B_{n}(x)$, Euler $E_{n}(x)$ and Genocchi $G_{n}(x)$ polynomial are defined by means of the following generating function as follows

$$
\frac{t}{e^{t}-1} e^{x t}=\sum_{n=0}^{\infty} B_{n}(x) \frac{t^{n}}{n !},|t|<2 \pi, \frac{2}{e^{t}+1} e^{x t}=\sum_{n=0}^{\infty} E_{n}(x) \frac{t^{n}}{n !},|t|<\pi,
$$

and

$$
\frac{2 t}{e^{t}+1} e^{x t}=\sum_{n=0}^{\infty} G_{n}(x) \frac{t^{n}}{n !},|t|<\pi,(\text { see }[1-25])
$$

respectively.

For $u \in \mathbb{C}$ with $u \neq 1$, the classical Frobenius-Euler polynomials $H_{n}^{(\alpha)}(x ; u)$ of order $\alpha$ are defined by means of the following generating function

$$
\left(\frac{1-u}{e^{t}-u}\right)^{\alpha} e^{x t}=\sum_{n=0}^{\infty} H_{n}^{(\alpha)}(x ; u) \frac{t^{n}}{n !},(\text { see }[1,5,11,12]) .
$$

In the special case when $x=0, H_{n}^{(\alpha)}(u)=H_{n}^{(\alpha)}(0 ; u)$ are called $n^{\text {th }}$ FrobeniusEuler numbers of order $\alpha$. For $\alpha=1$ into $(1.2), H_{n}^{(1)}(x, u)=H_{n}(x, u)$, are called the Frobenius-Euler polynomials and $H_{n}^{(\alpha)}(0 ; u)=h_{n}^{(\alpha)}(u)$, are called the Frobenius-Euler numbers of order $\alpha$. Substituting $u=-1$ into $(1.2), H_{n}(x ;-1)=E_{n}(x)$, are called the Euler polynomials, (see [8, 22, 24, 25]). by

In (2017), Kurt [10] introduced the poly-Frobenius-Euler polynomials are given

$$
\frac{(1-u) \operatorname{Li}_{k}\left(1-e^{-t}\right)}{t\left(e^{t}-u\right)} e^{x t}=\sum_{n=0}^{\infty} H_{n}^{(k)}(x ; u) \frac{t^{n}}{n !} .
$$


In the case when $x=0, H_{n}^{(k)}(u)=H_{n}^{(k)}(0 ; u)$ are called the poly-FrobeniusEuler numbers. by

For any non-zero $\lambda \in \mathbb{R}$ (or $\mathbb{C}$ ), the degenerate exponential function is defined

$$
e_{\lambda}^{x}(t)=(1+\lambda t)^{\frac{x}{\lambda}}, e_{\lambda}(t)=(1+\lambda t)^{\frac{1}{\lambda}},(\text { see }[13,18,19])
$$

By binomial expansion, we get

$$
e_{\lambda}^{x}(t)=\sum_{n=0}^{\infty}(x)_{n, \lambda} \frac{t^{n}}{n !},(\text { see }[14,17]),
$$

where $(x)_{0, \lambda}=1,(x)_{n, \lambda}=(x-\lambda)(x-2 \lambda) \cdots(x-(n-1) \lambda),(n \geq 1)$.

Note that

$$
\lim _{\lambda \longrightarrow 0} e_{\lambda}^{x}(t)=\sum_{n=0}^{\infty} x^{n} \frac{t^{n}}{n !}=e^{x t}
$$

In $[2,3]$ Carlitz introduced the degenerate Bernoulli and degenerate Euler polynomials are defined by

$$
\frac{z}{e_{\lambda}(z)-1} e_{\lambda}^{x}(z)=\sum_{j=0}^{\infty} B_{j, \lambda}(x) \frac{z^{j}}{j !}, \frac{2}{e_{\lambda}(z)+1} e_{\lambda}^{x}(z)=\sum_{j=0}^{\infty} E_{j, \lambda}(x) \frac{z^{j}}{j !},
$$

respectively.

In the case when $x=0, B_{j, \lambda}=B_{j, \lambda}(0)$ are called the degenerate Bernoulli numbers and $x=0, E_{j, \lambda}=E_{j, \lambda}(0)$ are called the degenerate Euler numbers.

Obviously

$$
\lim _{\lambda \longrightarrow 0} \beta_{n}(x ; \lambda)=B_{n}(x), \lim _{\lambda \longrightarrow 0} E_{n}(x ; \lambda)=E_{n}(x) .
$$

Kim et al. [15] introduced the degenerate Frobenius-Euler polynomials are defined by means of the generating function as follows

$$
\frac{1-u}{(1+\lambda t)^{\frac{1}{\lambda}}-u}(1+\lambda t)^{\frac{x}{\lambda}}=\sum_{n=0}^{\infty} h_{n, \lambda}(x \mid u) \frac{t^{n}}{n !},
$$

At the value $x=0, h_{n, \lambda}(u)=h_{n, \lambda}(0 \mid u)$ are called the degenerate FrobeniusEuler numbers.

It is readily seen that

$$
\lim _{\lambda \longrightarrow 0} h_{n, \lambda}(x \mid u)=H_{n}(x \mid u),(n \geq 0) .
$$

For $s \in \mathbb{Z}$, the polylogaritm function is defined by a power series in $\mathrm{z}$ as

$$
\operatorname{Li}_{s}(z)=\sum_{j=1}^{\infty} \frac{j^{n}}{j^{s}}=z+\frac{z^{2}}{2^{s}}+\frac{z^{3}}{3^{s}},(|z|<1),(\text { see },[4,9]) .
$$

It is notice that

$$
\operatorname{Li}_{1}(z)=\sum_{j=1}^{\infty} \frac{z^{j}}{j}=-\log (1-z)
$$


For $\lambda \in \mathbb{R}$, Kim-Kim [19] defined the degenerate version of the logarithm function, denoted by $\log _{\lambda}(1+z)$ as follows:

$$
\log _{\lambda}(1+z)=\sum_{j=1}^{\infty} \lambda^{j-1}(1)_{j, 1 / \lambda} \frac{z^{j}}{j !},(\text { see, }[18])
$$

being the inverse of the degenerate version of the exponential function $e_{\lambda}(z)$ as has been shown below

It is noteworthy to mention that

$$
e_{\lambda}\left(\log _{\lambda}(z)\right)=\log _{\lambda}\left(e_{\lambda}(z)\right)=z .
$$

$$
\lim _{\lambda \rightarrow 0} \log _{\lambda}(1+z)=\sum_{j=1}^{\infty}(-1)^{j-1} \frac{z^{j}}{j !}=\log (1+z) .
$$

The degenerate polylogarithm function [19] is defined by Kim-Kim to be

$$
l_{k, \lambda}(z)=\sum_{j=1}^{\infty} \frac{(-\lambda)^{j-1}(1)_{j, 1 / \lambda}}{(j-1) ! j^{k}} z^{n},(k \in \mathbb{Z},|z|<1) .
$$

It is clear that

$$
\lim _{\lambda \rightarrow 0} l_{k, \lambda}(z)=\sum_{j=1}^{\infty} \frac{z^{j}}{j^{k}}=\operatorname{Li}_{k}(z),(\text { see }[4,9]) .
$$

From (1.10) and (1.11), we get

$$
l_{1, \lambda}(z)=\sum_{j=1}^{\infty} \frac{(-\lambda)^{j-1}(1)_{j, 1 / \lambda}}{j !} z^{j}=-\log _{\lambda}(1-z) .
$$

Very recently, Kim-Kim [19] introduced the new type degenerate version of the Bernoulli polynomials and numbers, by using the degenerate polylogarithm function as follows

$$
\frac{l_{k, \lambda}\left(1-e_{\lambda}(-z)\right)}{1-e_{\lambda}(-z)} e_{\lambda}^{u}(z)=\sum_{j=0}^{\infty} \beta_{j, \lambda}^{(k)}(u) \frac{z^{j}}{j !} .
$$
numbers.

In the special case $x=0, \beta_{j, \lambda}^{(k)}=\beta_{j, \lambda}^{(k)}(0)$ are called the degenerate poly-Bernoulli

It is well known that the Stirling numbers of the first kind are defined by

$$
(x)_{n}=\sum_{l=0}^{n} S_{1}(n, l) x^{l},(\text { see }[24,25]),
$$

where $(x)_{0}=1$, and $(x)_{n}=x(x-1) \cdots(x-n+1),(n \geq 1)$. From (1.13), it is easily to see that

$$
\frac{1}{k !}(\log (1+t))^{k}=\sum_{n=k}^{\infty} S_{1}(n, k) \frac{t^{n}}{n !},(k \geq 0),(\text { see }[12,13,18]) .
$$

In the inverse expression to (1.14), the Stirling numbers of the second kind are defined by

$$
x^{n}=\sum_{l=0}^{n} S_{2}(n, l)(x)_{l},(\text { see }[10,12,22]) .
$$

From (1.15), it is easily to see that

$$
\frac{1}{k !}\left(e^{t}-1\right)^{k}=\sum_{n=l}^{\infty} S_{2}(n, l) \frac{t^{n}}{n !},(\text { see }[22,24,25]) .
$$


For $n \geq 0$, the degenerate Stirling numbers of the second kind [7, 8, 17] are defined by

$$
\frac{1}{n !}\left(e_{\lambda}(t)-1\right)^{n}=\sum_{l=n}^{\infty} S_{2, \lambda}(l, n) \frac{t^{l}}{l !},(n \geq 0) .
$$

In this paper, we construct the degenerate poly-Frobenius-Euler polynomials and numbers, called the new type of poly-Frobenius-Euler polynomials and numbers by using the degenerate polylogarithm function and derive several properties on the degenerate poly-Frobenius-Euler polynomials and numbers.

\section{New type of degenerate poly-Frobenius-Euler polynomials}

Let $\lambda, u \in \mathbb{C}$ with $u \neq 1$ and $k \in \mathbb{Z}$, by using the degenerate polylogarithm function, we define the new type of degenerate poly-Frobenius-Euler polynomials as follows

$$
\frac{(1-u) l_{k, \lambda}\left(1-e_{\lambda}(-t)\right)}{t\left(e_{\lambda}(t)-u\right)} e_{\lambda}^{x}(t)=\sum_{n=0}^{\infty} H_{n, \lambda}^{(k)}(x ; u) \frac{t^{n}}{n !}
$$

In the special case, $x=0, H_{n, \lambda}^{(k)}(u)=H_{n, \lambda}^{(k)}(0 ; u)$ are called the new type of degenerate poly-Frobenius-Euler numbers.

For $k=1$ in $(2.1)$, we get

$$
\frac{1-u}{e_{\lambda}(t)-u} e_{\lambda}^{x}(t)=\sum_{n=0}^{\infty} h_{n, \lambda}(x ; u) \frac{t^{n}}{n !},(\text { see }[15])
$$

where $h_{n, \lambda}(x ; u)$ are called the degenerate Frobenius-Euler polynomials.

Theorem 2.1. For $n \geq 0$, we have

$$
H_{n, \lambda}^{(k)}(x ; u)=\sum_{m=0}^{n}\left(\begin{array}{c}
n \\
m
\end{array}\right) H_{n-m, \lambda}^{(k)}(u)(x)_{m, \lambda} .
$$

Proof. From (2.1), we have

$$
\begin{gathered}
\sum_{n=0}^{\infty} H_{n, \lambda}^{(k)}(x ; u) \frac{t^{n}}{n !}=\left(\frac{(1-u) l_{k, \lambda}\left(1-e_{\lambda}(-t)\right)}{t\left(e_{\lambda}(t)-u\right)}\right) e_{\lambda}^{x}(t) \\
=\sum_{n=0}^{\infty} H_{n, \lambda}^{(k)}(u) \frac{t^{n}}{n !} \sum_{m=0}^{\infty}(x)_{m, \lambda} \frac{t^{m}}{m !} \\
\text { L.H.S }=\sum_{n=0}^{\infty} \sum_{m=0}^{n}\left(\begin{array}{c}
n \\
m
\end{array}\right) H_{n-m, \lambda}^{(k)}(u)(x)_{m, \lambda} \frac{t^{n}}{n !} .
\end{gathered}
$$

Therefore, by (2.1) and (2.3), we require at the desired result.

Theorem 2.2. For $k \in \mathbb{Z}$ and $n \geq 0$, we have

$$
H_{n, \lambda}^{(k)}(x ; u)=\sum_{l=0}^{n}\left(\begin{array}{l}
n \\
l
\end{array}\right) \sum_{m=0}^{l} \frac{(1)_{m+1,1 / \lambda}(-\lambda)^{m}(-1)^{l-m}}{(m+1)^{k-1}} \frac{S_{2, \lambda}(l+1, m+1)}{l+1} h_{n-l, \lambda}(x ; u) .
$$


Proof. It is proved by using (1.7), ( 1.11) and (2.1) that

$$
\begin{gathered}
\sum_{n=0}^{\infty} H_{n, \lambda}^{(k)}(x ; u) \frac{t^{n}}{n !}=\left(\frac{(1-u) e_{\lambda}^{x}(t)}{t\left(e_{\lambda}(t)-u\right)} l_{k, \lambda}\left(1-e_{\lambda}(-t)\right)\right. \\
=\left(\frac{(1-u) e_{\lambda}^{x}(t)}{t\left(e_{\lambda}(t)-u\right)}\right) \sum_{m=1}^{\infty} \frac{(1)_{m, 1 / \lambda}(-\lambda)^{m-1}}{(m-1) ! m^{k}}\left(1-e_{\lambda}(-t)\right)^{m} \\
=\left(\frac{(1-u) e_{\lambda}^{x}(t)}{t\left(e_{\lambda}(t)-u\right)}\right) \sum_{m=0}^{\infty} \frac{(1)_{m+1,1 / \lambda}(-\lambda)^{m}}{(m+1)^{k-1} m !}\left(1-e_{\lambda}(-t)\right)^{m+1} \\
=\left(\frac{(1-u) e_{\lambda}^{x}(t)}{t\left(e_{\lambda}(t)-u\right)}\right) \sum_{m=0}^{\infty} \frac{(1)_{m+1,1 / \lambda}(-\lambda)^{m}}{(m+1)^{k-1}} \sum_{l=m+1}^{\infty} S_{2, \lambda}(l, m+1)(-1)^{l-m-1} \frac{t^{l}}{l !} \\
=\left(\frac{(1-u) e_{\lambda}^{x}(t)}{e_{\lambda}(t)-u}\right) \sum_{l=0}^{\infty} \sum_{m=0}^{l} \frac{(1)_{m+1,1 / \lambda}(-\lambda)^{m}(-1)^{l-m}}{(m+1)^{k-1}} \frac{S_{2, \lambda}(l+1, m+1)}{l+1} \frac{t^{l}}{l !} \\
=\sum_{n=0}^{\infty} h_{n, \lambda}(x ; u) \frac{t^{n}}{n !} \sum_{l=0}^{\infty} \sum_{m=0}^{l} \frac{(1)_{m+1,1 / \lambda}(-\lambda)^{m}(-1)^{l-m}}{(m+1)^{k-1}} \frac{S_{2, \lambda}(l+1, m+1)}{l+1} \frac{t^{l}}{l !} \\
\text { L.H.S = } \sum_{n=0}^{\infty}\left(\sum_{l=0}^{n}\left(\begin{array}{l}
n \\
l
\end{array}\right) \sum_{m=0}^{l} \frac{(1)_{m+1,1 / \lambda}(-\lambda)^{m}(-1)^{l-m}}{(m+1)^{k-1}} \frac{S_{2, \lambda}(l+1, m+1)}{l+1} h_{n-l, \lambda}(x ; u)\right) \frac{t^{n}}{n !} .
\end{gathered}
$$

By comparing the coefficients of $\frac{t^{n}}{n !}$ on both sides, we get the result.

Corollary 2.1. For $k \in \mathbb{Z}$ and $n \geq 0$, we have

$$
H_{n, \lambda}^{(k)}(u)=\sum_{l=0}^{n}\left(\begin{array}{l}
n \\
l
\end{array}\right) \sum_{m=0}^{l} \frac{(1)_{m+1,1 / \lambda}(-\lambda)^{m}(-1)^{l-m}}{(m+1)^{k-1}} \frac{S_{2, \lambda}(l+1, m+1)}{l+1} h_{n-l, \lambda}(u) .
$$

Corollary 2.2. For $n \geq 0$, we have

$$
H_{n, \lambda}(x ; u)=\sum_{l=0}^{n}\left(\begin{array}{l}
n \\
l
\end{array}\right) \sum_{m=0}^{l} \frac{(1)_{m+1,1 / \lambda}(-\lambda)^{m}(-1)^{l-m} S_{2, \lambda}(l+1, m+1)}{l+1} h_{n-l, \lambda}(x ; u) .
$$

Corollary 2.3. For $n \geq 0$, we have

$$
E_{n, \lambda}(x)=\sum_{l=0}^{n}\left(\begin{array}{l}
n \\
l
\end{array}\right) \sum_{m=0}^{l} \frac{(1)_{m+1,1 / \lambda}(-\lambda)^{m}(-1)^{l-m} S_{2, \lambda}(l+1, m+1)}{l+1} E_{n-l, \lambda}(x) .
$$

Moreover,

$$
\sum_{l=0}^{n}\left(\begin{array}{l}
n \\
l
\end{array}\right) \sum_{m=0}^{l} \frac{(1)_{m+1,1 / \lambda}(-\lambda)^{m}(-1)^{l-m} S_{2, \lambda}(l+1, m+1)}{l+1} E_{n-l, \lambda}(x)=0 .
$$

Theorem 2.3. For $n \geq 0$, we have

$$
\begin{gathered}
\sum_{l=0}^{n}\left(\begin{array}{l}
n \\
l
\end{array}\right) \sum_{m=0}^{l} \frac{(1)_{m+1,1 / \lambda}(-\lambda)^{m}(-1)^{l-m}}{(m+1)^{k-1}} \frac{S_{2, \lambda}(l+1, m+1)}{l+1}(x)_{n-l, \lambda} \\
=\frac{1}{1-u}\left[\sum_{m=0}^{n}\left(\begin{array}{c}
n \\
m
\end{array}\right) H_{n-m, \lambda}^{(k)}(x ; u)(1)_{m, \lambda}-u H_{n, \lambda}^{(k)}(x ; u)\right] .
\end{gathered}
$$


Proof. From (2.1), we have

$$
\begin{gathered}
\frac{(1-u) l_{k, \lambda}\left(1-e_{\lambda}(-t)\right)}{t} e_{\lambda}^{x}(t)=e_{\lambda}(t) \sum_{n=0}^{\infty} H_{n, \lambda}^{(k)}(x ; u) \frac{t^{n}}{n !}-u \sum_{n=0}^{\infty} H_{n, \lambda}^{(k)}(x ; u) \frac{t^{n}}{n !} \\
=\sum_{m=0}^{\infty}(1)_{m, \lambda} \frac{t^{m}}{m !} \sum_{n=0}^{\infty} H_{n, \lambda}^{(k)}(x ; u) \frac{t^{n}}{n !}-u \sum_{n=0}^{\infty} H_{n, \lambda}^{(k)}(x ; u) \frac{t^{n}}{n !} \\
=\sum_{n=0}^{\infty}\left(\sum_{m=0}^{n}\left(\begin{array}{c}
n \\
m
\end{array}\right) H_{n-m, \lambda}^{(k)}(x ; u)(1)_{m, \lambda}-u H_{n, \lambda}^{(k)}(x ; u)\right) \frac{t^{n}}{n !}
\end{gathered}
$$

On the other hand,

$$
\begin{gathered}
\frac{(1-u) l_{k, \lambda}\left(1-e_{\lambda}(-t)\right)}{t} e_{\lambda}^{x}(t) \\
=\left(\sum_{n=0}^{\infty}(x)_{n, \lambda} \frac{t^{n}}{n !}\right) \frac{(1-u)}{t}\left(\sum_{m=1}^{\infty} \frac{(1)_{m, 1 / \lambda}(-\lambda)^{m-1}}{(m-1) ! m^{k}}\left(1-e_{\lambda}(-t)\right)^{m}\right) \\
=\left(\sum_{n=0}^{\infty}(x)_{n, \lambda} \frac{t^{n}}{n !}\right) \frac{(1-u)}{t}\left(\sum_{m=0}^{\infty} \frac{(1)_{m+1,1 / \lambda}(-\lambda)^{m}}{(m+1)^{k-1} m !}\left(1-e_{\lambda}(-t)\right)^{m+1}\right) \\
=(1-u)\left(\sum_{n=0}^{\infty}(x)_{n, \lambda} \frac{t^{n}}{n !}\right)\left(\sum_{l=0}^{\infty} \sum_{m=0}^{l} \frac{(1)_{m+1,1 / \lambda}(-\lambda)^{m}(-1)^{l-m}}{(m+1)^{k-1}} \frac{S_{2, \lambda}(l+1, m+1)}{l+1} \frac{t^{l}}{l !}\right) \\
\text { L.H.S }=(1-u) \sum_{n=0}^{\infty}\left(\sum_{l=0}^{n}\left(\begin{array}{l}
n \\
l
\end{array}\right) \sum_{m=0}^{l} \frac{(1)_{m+1,1 / \lambda}(-\lambda)^{m}(-1)^{l-m}}{(m+1)^{k-1}} \frac{S_{2, \lambda}(l+1, m+1)}{l+1}(x)_{n-l, \lambda}\right) \frac{t^{n}}{n !} .
\end{gathered}
$$

Comparing the coefficients of $\frac{t^{n}}{n !}$ on both sides, we get the result.

Theorem 2.4. For $n \geq 0$, we have

$$
H_{n, \lambda}^{(k)}(u)=\frac{(1-u)}{x\left(e_{\lambda}(x)-u\right)} \int_{0}^{x} \underbrace{\frac{e_{\lambda}^{1-\lambda}(-t)}{1-e_{\lambda}(-t)} \int_{0}^{t} \frac{e_{\lambda}^{1-\lambda}(-t)}{1-e_{\lambda}(-t)} \cdots \int_{0}^{t} \frac{e_{\lambda}^{1-\lambda}(-t)}{1-e_{\lambda}(-t)}}_{(k-2)-\text { times }} t d t d t \cdots d t .
$$

Proof. Using (1.11), we first consider the following expression

$$
\begin{aligned}
\frac{d}{d x} l_{k, \lambda}\left(1-e_{\lambda}(-x)\right)= & \frac{d}{d x} \sum_{n=1}^{\infty} \frac{(1)_{n, 1 / \lambda}}{(n+1) ! n^{k}}\left(1-e_{\lambda}(-x)\right)^{n} \\
& =\frac{1}{1-e_{\lambda}(-x)} l_{k-1, \lambda}\left(1-e_{\lambda}(-x)\right) .
\end{aligned}
$$

From (2.7), $k \geq 2$, we have

$$
\begin{gathered}
\sum_{n=0}^{\infty} H_{n, \lambda}^{(k)}(u) \frac{x^{n}}{n !}=\frac{(1-u)}{x\left(e_{\lambda}(x)-u\right)} l_{k, \lambda}\left(1-e_{\lambda}(-x)\right) \\
=\frac{(1-u)}{x\left(e_{\lambda}(x)-u\right)} \int_{0}^{x} \frac{e_{\lambda}^{1-\lambda}(-t)}{1-e_{\lambda}(-t)} \int_{0}^{t} \frac{e_{\lambda}^{1-\lambda}(-t)}{1-e_{\lambda}(-t)} \cdots \int_{0}^{t} \frac{e_{\lambda}^{1-\lambda}(-t)}{1-e_{\lambda}(-t)} t d t d t \cdots d t \\
=\frac{(1-u)}{x\left(e_{\lambda}(x)-u\right)} \int_{0}^{x} \underbrace{\frac{e_{\lambda}^{1-\lambda}(-t)}{1-e_{\lambda}(-t)} \int_{0}^{t} \frac{e_{\lambda}^{1-\lambda}(-t)}{1-e_{\lambda}(-t)} \cdots \int_{0}^{t} \frac{e_{\lambda}^{1-\lambda}(-t)}{1-e_{\lambda}(-t)} t d t d t \cdots d t .}_{(k-2)-\text { times }}
\end{gathered}
$$


By (2.9), we obtain at the desired result. Thus, we complete the proof.

Theorem 2.5. Let $n \geq 0$. Then

$H_{n, \lambda}^{(2)}(u)=\sum_{m=0}^{n}\left(\begin{array}{c}n \\ m\end{array}\right)(-1)^{m} \frac{B_{m, \lambda}(1-\lambda)}{m+1} h_{n-m, \lambda}(u)=\sum_{m=0}^{n}\left(\begin{array}{c}n \\ m\end{array}\right)(-1)^{n-m} \frac{B_{n-m, \lambda}(1-\lambda)}{n-m+1} h_{m, \lambda}(u)$.

Proof. By using (1.17) and Theorem 2.4, we get

$$
\begin{gathered}
\sum_{n=0}^{\infty} H_{n, \lambda}^{(2)}(u) \frac{x^{n}}{n !}=\frac{1-u}{x\left(e_{\lambda}(x)-u\right)} \int_{0}^{x} \frac{-t}{e_{\lambda}(-t)-1} e_{\lambda}^{1-\lambda}(-t) d t \\
=\frac{1-u}{x\left(e_{\lambda}(x)-u\right)} \int_{0}^{x} \sum_{n=0}^{\infty} B_{n, \lambda}(1-\lambda) \frac{(-t)^{n}}{n !} d t \\
=\frac{1-u}{x\left(e_{\lambda}(x)-u\right)} \sum_{m=0}^{\infty}(-1)^{m} \frac{B_{m, \lambda}(1-\lambda)}{m+1} \frac{x^{m}}{m !} \\
=\left(\sum_{n=0}^{\infty} h_{n, \lambda}(u) \frac{x^{n}}{n !}\right)\left(\sum_{m=0}^{\infty}(-1)^{m} \frac{B_{m, \lambda}(1-\lambda)}{m+1} \frac{x^{m}}{m !}\right) \\
\text { L.H.S }=\sum_{n=0}^{\infty}\left(\sum_{m=0}^{n}\left(\begin{array}{c}
n \\
m
\end{array}\right)(-1)^{m} \frac{B_{m, \lambda}(1-\lambda)}{m+1} h_{n-m, \lambda}(u)\right) \frac{x^{n}}{n !} .
\end{gathered}
$$

Therefore, by (2.10), we get the following theorem.

Theorem 2.6. Let $k \in \mathbb{Z}$ and $n \geq 0$, we have

$$
\begin{gathered}
H_{n, \lambda}^{(k)}(u)=\sum_{m=0}^{n}\left(\begin{array}{c}
n \\
m
\end{array}\right) \sum_{m_{1}+m_{2}+\cdots+m_{k-1}=m}\left(\begin{array}{c}
m \\
m_{1}, m_{2}, \cdots, m_{k}
\end{array}\right) h_{n-m \cdot \lambda}(u) \\
\times \frac{\beta_{m_{1}, \lambda}(\lambda-1)}{m_{1}+1} \frac{\beta_{m_{2}, \lambda}(\lambda-1)}{m_{2}+m_{2}+1} \cdots \frac{\beta_{m_{k-1}, \lambda}(\lambda-1)}{m_{1}+m_{2}+\cdots+n_{k-1}+1} .
\end{gathered}
$$

Proof. In general, from (2.9), we note that

$$
\begin{gathered}
\sum_{n=0}^{\infty} H_{n, \lambda}^{(k)}(u) \frac{x^{n}}{n !}=\frac{1-u}{x\left(e_{\lambda}(x)-u\right)} \int_{0}^{x} \underbrace{\frac{e_{\lambda}^{1-\lambda}(-t)}{1-e_{\lambda}(-t)} \int_{0}^{t} \frac{e_{\lambda}^{1-\lambda}(-t)}{1-e_{\lambda}(-t)} \cdots \int_{0}^{t} \frac{e_{\lambda}^{1-\lambda}(-t)}{1-e_{\lambda}(-t)} t d t d t \cdots d t}_{(k-2)-\text { times }} \\
=\frac{1-u}{e_{\lambda}(x)-u} \sum_{m=0}^{\infty} \sum_{m_{1}+m_{2}+\cdots+m_{k-1}=m}^{\infty}\left(\begin{array}{c}
m \\
m_{1}, m_{2}, \cdots, m_{k}
\end{array}\right) \frac{\beta_{m_{1}, \lambda}(\lambda-1)}{m_{1}+1} \frac{\beta_{m_{2}, \lambda}(\lambda-1)}{m_{2}+m_{2}+1} \\
\quad \times \cdots \frac{\beta_{m_{k-1}, \lambda}(\lambda-1)}{m_{1}+m_{2}+\cdots+n_{k-1}+1} \frac{x^{m}}{m !} \\
L . H . S=\sum_{n=0}^{\infty}\left(\sum_{m=0}^{n}\left(\begin{array}{c}
n \\
m
\end{array}\right) \sum_{m_{1}+m_{2}+\cdots+m_{k-1}=m}\left(\begin{array}{c}
m \\
m_{1}, m_{2}, \cdots, m_{k}
\end{array}\right) h_{n-m . \lambda}(u)\right. \\
\left.\quad \times \frac{\beta_{m_{1}, \lambda}(\lambda-1)}{m_{1}+1} \frac{\beta_{m_{2}, \lambda}(\lambda-1)}{m_{2}+m_{2}+1} \cdots \frac{\beta_{m_{k-1}, \lambda}(\lambda-1)}{m_{1}+m_{2}+\cdots+n_{k-1}+1}\right) \frac{x^{n}}{n !} .
\end{gathered}
$$
result.

Therefore, by comparing the coefficients of $t^{n}$ on both sides, we obtain the 
Theorem 2.7. For $n \geq 0$, we have

$$
H_{n, \lambda}^{(k)}(x+y ; u)=\sum_{m=0}^{n}\left(\begin{array}{c}
n \\
m
\end{array}\right) H_{n-m, \lambda}^{(k)}(x ; u)(y)_{m, \lambda} .
$$

Proof. From (2.1), we have

$$
\begin{gathered}
\sum_{n=0}^{\infty} H_{n, \lambda}^{(k)}(x+y ; u) \frac{t^{n}}{n !}=\left(\frac{(1-u) l_{k, \lambda}\left(1-e_{\lambda}(-t)\right)}{t\left(e_{\lambda}(t)-u\right)}\right) e_{\lambda}^{x+y}(t) \\
=\left(\sum_{n=0}^{\infty} H_{n, \lambda}^{(k)}(x ; u) \frac{t^{n}}{n !}\right)\left(\sum_{m=0}^{\infty}(y)_{m, \lambda} \frac{t^{m}}{m !}\right) \\
\text { L.H.S }=\sum_{n=0}^{\infty}\left(\sum_{m=0}^{n}\left(\begin{array}{c}
n \\
m
\end{array}\right) H_{n-m, \lambda}^{(k)}(x ; u)(y)_{m, \lambda}\right) \frac{t^{n}}{n !} .
\end{gathered}
$$

Comparing the coefficients on both sides of (2.12), we get the result.

Theorem 2.8. For $n \geq 0$, we have

$$
H_{n, \lambda}^{(k)}(x+1 ; u)=\sum_{m=0}^{n}\left(\begin{array}{c}
n \\
m
\end{array}\right) H_{n-m, \lambda}^{(k)}(x ; u)(1)_{m, \lambda} .
$$

Proof. By (2.1), we observe that

$$
\begin{gathered}
\sum_{n=0}^{\infty}\left[H_{n, \lambda}^{(k)}(x+1 ; u)-H_{n, \lambda}^{(k)}(x ; u)\right] \frac{t^{n}}{n !}=\left(\frac{(1-u) l_{k, \lambda}\left(1-e_{\lambda}(-t)\right)}{t\left(e_{\lambda}(t)-u\right)}\right) e_{\lambda}^{x}(t)\left[e_{\lambda}(t)-1\right] \\
\text { L.H.S }=\sum_{n=0}^{\infty} \sum_{m=0}^{n}\left(\begin{array}{c}
n \\
m
\end{array}\right) H_{n-m, \lambda}^{(k)}(x ; u)(1)_{m, \lambda} \frac{t^{n}}{n !}-\sum_{n=0}^{\infty} H_{n, \lambda}^{(k)}(x ; u) \frac{t^{n}}{n !} \\
\text { Comparing the coefficients of } t^{n} \text { on both sides, we obtain the result. }
\end{gathered}
$$

Theorem 2.9. For $n \geq 0$, we have

$$
H_{n, \lambda}^{(k)}(x ; u)=\sum_{m=0}^{n} \sum_{q=0}^{m}\left(\begin{array}{l}
n \\
m
\end{array}\right)(x)_{q} S_{\lambda}^{(2)}(m, q) H_{n-m, \lambda}^{(k)}(u) .
$$

Proof. From (2.1), we have

$$
\begin{gathered}
\sum_{n=0}^{\infty} H_{n, \lambda}^{(k)}(x ; u) \frac{t^{n}}{n !}=\left(\frac{(1-u) l_{k, \lambda}\left(1-e_{\lambda}(-x)\right)}{t\left(e_{\lambda}(t)-u\right)}\right) e_{\lambda}^{x}(t) \\
=\left(\frac{(1-u) l_{k, \lambda}\left(1-e_{\lambda}(-x)\right)}{t\left(e_{\lambda}(t)-u\right)}\right)\left[e_{\lambda}(t)-1+1\right]^{x} \\
=\left(\frac{(1-u) l_{k, \lambda}\left(1-e_{\lambda}(-x)\right)}{t\left(e_{\lambda}(t)-u\right)}\right)\left(\sum_{q=0}^{\infty}(x)_{q} \sum_{l=q}^{\infty} S_{\lambda}^{(2)}(l, q) \frac{t^{l}}{l !}\right) \\
\text { L.H.S }=\sum_{n=0}^{\infty}\left(\sum_{m=0}^{n} \sum_{q=0}^{m}\left(\begin{array}{c}
n \\
m
\end{array}\right)(x)_{q} S_{\lambda}^{(2)}(m, q) H_{n-m, \lambda}^{(k)}(u)\right) \frac{t^{n}}{n !} .
\end{gathered}
$$

By comparing the coefficients of $t^{n}$ on both sides, we get the result.

Theorem 2.10. For $n \geq 0$, we have

$$
H_{n, \lambda}^{(k)}(x+\alpha \mid u)=\sum_{l=0}^{n} \sum_{m=0}^{l}\left(\begin{array}{c}
n \\
l
\end{array}\right) x^{m} m ! S_{2, \lambda}(l+\alpha, m+\alpha) H_{n-l, \lambda}^{(k)}(u) .
$$


Proof. Replacing $x$ by $x+\alpha$ in (2.1), we have

$$
\begin{aligned}
& \sum_{n=0}^{\infty} H_{n, \lambda}^{(k)}(x+\alpha ; u) \frac{t^{n}}{n !}=\left(\frac{(1-u) l_{k, \lambda}\left(1-e_{\lambda}(-x)\right)}{t\left(e_{\lambda}(t)-u\right)}\right) e_{\lambda}^{x+\alpha}(t) \\
= & \left(\frac{(1-u) l_{k, \lambda}\left(1-e_{\lambda}(-x)\right)}{t\left(e_{\lambda}(t)-u\right)}\right) e_{\lambda}^{\alpha}(t)\left(\sum_{m=0}^{\infty} x^{m}\left(e_{\lambda}(t)-1\right)^{m}\right) \\
= & \left(\frac{(1-u) l_{k, \lambda}\left(1-e_{\lambda}(-x)\right)}{t\left(e_{\lambda}(t)-u\right)}\right)\left(e_{\lambda}^{\alpha}(t) \sum_{m=0}^{\infty} x^{m} m ! \frac{\left(e_{\lambda}(t)-1\right)^{m}}{m !}\right) \\
= & \left(\frac{(1-u) l_{k, \lambda}\left(1-e_{\lambda}(-x)\right)}{t\left(e_{\lambda}(t)-u\right)}\right)\left(e_{\lambda}^{\alpha}(t) \sum_{m=0}^{\infty} x^{m} m ! \sum_{l=m}^{\infty} S_{2, \lambda}(l, m) \frac{t^{l}}{l !}\right) \\
= & \sum_{n=0}^{\infty} H_{n, \lambda}^{(k)}(u) \frac{t^{n}}{n !}\left(\sum_{l=0}^{\infty} \sum_{m=0}^{l} x^{m} m ! S_{2, \lambda}(l+\alpha, m+\alpha) \frac{t^{l}}{l !}\right) \\
\text { L.H.S }= & \sum_{n=0}^{\infty}\left(\sum_{l=0}^{n} \sum_{m=0}^{l}\left(\begin{array}{c}
n \\
l
\end{array}\right) x^{m} m ! S_{2, \lambda}(l+\alpha, m+\alpha) H_{n-l, \lambda}^{(k)}(u)\right) \frac{t^{n}}{n !} .
\end{aligned}
$$

Therefore, by (2.1) and (2.15), we obtain the result.

\section{Conclusions}

Motivated by the definition of the degenerate poly-Bernoulli polynomials introduced by Kim-Kim [19], in the present paper, we have considered a class of new generating function for the degenerate Frobenius-Euler polynomials, called the new type of degenerate poly-Frobenius-Euler polynomials, by means of the degenerate polylogarithm function. Then, we have derived some useful relations and properties. We have showed that the new type of degenerate poly-Frobenius-Euler polynomials equal a linear combination of the degenerate Frobenius-Euler polynomials and Stirlings numbers of the first and second kind. In a special case, we have given a relation between the new type of degenerate Frobenius-Euler polynomials and Bernoulli polynomials of order $n$.

Author Contributions: All authors contributed equally to the manuscript and typed, read, and approved final manuscript.

Conflict of Interest: The authors declare no conflict of interest.

Funding: None.

Acknowledgements: None.

\section{References}

[1] Araci, S.; Acikgoz, M. A note on the Frobenius-Euler numbers and polynomials associated with Bernstein polynomials. Adv. Stud. Contemp. Math. 2012, 22(3), 399-406.

[2] Carlitz, L. Degenerate Stirling, Bernoulli and Eulerian numbers. Utilitas Math. 1979, 15, 51-88. 
[3] Carlitz, L. A degenerate Staudt-Clausen theorem, Arch. Math. (Basel) 1956, 7, 28-33.

[4] Eastham, M.S.P. On Polylogarithms. Proc. Glasgow Math. Assoc. 1964, 6, 169-171.

[5] Janson, S, Frobenius numbers and rounding, Reprint (2013), arXiv:1305.3512.

[6] Khan, W.A.; Srivastava, D. On the generalized Apostol type FrobeniusGenocchi polynomials. Filomat. 2019, 33(7), 1967-1977.

[7] Khan, W.A.; Ahmad, M. Partially degenerate poly-Bernoulli polynomials. Adv. Stud. Contemp. Math., 2018, 28(3), 487-496.

[8] Khan, W.A. A new class of degenerate Frobenius-Euler Hermite polynomials. Adv. Stud. Contemp. Math., 2018, 30(4), 567-576.

[9] Kaneko, M. Poly-Bernoulli numbers. J. Théor Nombres Bordeaux. 1997, 9(1), 221-228.

[10] Kurt, B. Poly-Frobenius-Euler polynomials, Proceedings of the International Conferences on Numerical Analysis and Applied Mathematics. Amer, Inst. Phys. Conf. Proc. 2016, 1863, 300019-1-300019-3.

[11] Kurt, B,; Simsek, Y. On the generalized Apostol type Frobenius Euler polynomials. Advances in Differences equations. 2013, 1-9.

[12] Kurt, B,; Simsek, Y. Frobenius Euler type polynomials related to HermiteBernoulli polynomials. Proceedings of the International Conferences on $\mathrm{Nu}-$ merical Analysis and Applied Mathematics, Amer, Inst. Phys. Conf. Proc. 2011, 1389, 385-388.

[13] Kim, D.S.; Kim, T. A note on polyexponential and unipoly functions. Russ. J. Math. Phys. bf 2019, 26(1), 40-49.

[14] Kim, T.; Kim, D.S. An identity of symmetry for the degenerate FrobeniusEuler polynomials. Math. Slovaca. 2018, 68(1), 239-243.

[15] Kim, T.; Kwon, H.I.; Seo, J.J. On the degenerate Frobenius-Euler polynomials. arXiv:1507.04846v1 [math. N.T] 17 July 2015.

[16] Kim, D.S.; Kim, T.; Seo, J.J. Higher-order Bernoulli, Frobenius-Euler and Euler polynomials, J. Comput. Anal. Appl. 2014, 17(1), 147-155.

[17] Kim, T. A note on degenerate Stirling polynomials of the second kind. Proc. Jangjeon Math. Soc. 2017, 20(3), 319-331.

[18] Kim, T.; Kim, D.S. Degenerate polyexponential functions and degenerate Bell polynomials. J. Math. Anal. Appl. 2020, 487(2), 124017.

[19] Kim, D.S.; Kim, T. A note on a new type of degenerate Bernoulli numbers. Russ. J. Math. Phys. 2020, 27(2), 227-235.

[18] Kim, T Kim, D.S.; Kim, H.-Y.; Jang, L.C. Degenerate poly-Bernoulli numbers and polynomials, Informatica. 3(13) (2020), 2-8.

[19] Kim, D.S.; Kim, T.; Komatsu, T.; Kwon, H.I. Barnes multiple Bernoulli and generalized Barnes multiple Frobenius-Euler mixed-type polynomials. Adv. Difference Equ. 2014, 2014:238.

[20] Ozden, H.; Cangul, I.N.; Simsek, Y. Remarks on $q$-Bernoulli numbers associated with Daehee numbers. Adv. Stud. Contemp. Math. (Kyungshang). 2009, 18(1), 41-48.

[21] Roman, S. The umbral calculus. Pure App. Math. Vol. 111. Academic Press, Inc., New York, 1984.

[22] Ryoo, C.S. A note on the Frobenius-Euler polynomials. Proc. Jangjeon Math. Soc. 2011, 14(4), 495-510.

[23] Simsek, Y. Identities on the Changhee numbers and Apostol-type Daehee polynomial. Adv. Stud. Contemp. Math. (Kyungshang). 2017, 27(2), 199-212. 
[24] Simsek, Y. Generating functions for $q$-Apostol type Frobenius-Euler numbers and polynomials. Axioms, 2012, 1, 395-403.

[25] Simsek, Y. Generating functions for generalized Stirlings type numbers array type polynomials, Eulerian type polynomials and their application. Fixed Point Theory and Appl. doi:10.1186/1687-1812-2013-87, 2013. 